\title{
Detección precoz del cáncer de cuello uterino mediante colposcopia Informe de 559 casos
}

\author{
G.E. Olarte*; G.R. Arango*; G.G. López*; E.V. Jaramillo*; S.L. Betancourt*; M.S. Trujillo*; M.O. Urrego**; \\ M.P. Vanegas** y M.T.G. Vallejo**
}

\begin{abstract}
RESUMEN: Con el propósito de efectuar diagnóstico precoz y tratamiento del cáncer de cuello uterino en el Departamento de Caldas, Colombia, S.A., se creó un grupo interdisciplinario o institucional descentralizado, que desde junio de 1989 se desplaza periódicamente y en forma continua a los Hospitales Regionales del Departamento de Caldas, para hacer actividades de prevención, educación a población de riesgo, adolescentes y detección del cáncer del cuello uterino. El ginecólogo practica colposcopia a todas las mujeres con citología anormal compatible con NIC o infección por VPH. Entre junio de 1989 y junio de 1992, se realizaron 559 colposcopias de primera vez; se diagnosticaron 152 casos de NIC y 21 casos de cáncer avanzado. La edad promedio para Ca. microinvasivo e invasivo es de 47.5 años; de 33.9 años para lesiones preinvasoras. $\mathrm{El} \mathrm{79.5 \%} \mathrm{de} \mathrm{mujeres} \mathrm{tuvo} \mathrm{relaciones} \mathrm{sexuales} \mathrm{antes} \mathrm{de} \mathrm{los} 20$ años y en la colposcopia se encontraron lesiones en el $60 \%$ de los casos, siendo el punteado y la zona acetoblanca los cambios más frecuentes. En 41 colposcopia se observaron vasos atípicos o lesiones clínicamente invasoras. De 404 biopsias tomadas se detectaron 21 carcinomas de tipo escamocelular, 4 microinvasores y 17 invasores.
\end{abstract}

El $88 \%$ de las lesiones fueron intraepiteliales. La sensibilidad de la colposcopia frente a la biopsia fue de $94 \%$ y la especificidad del $46 \%$. El $97 \%$ de los pacientes con PAP compatible con NIC-CA, presentaron algún tipo de lesión col poscópica. El promedio de edad de los pacient es con NIC fue 13.8 años menor que los pacientes con carcinoma invasor.

El $100 \%$ de la población estudiada es de clase económica baja y $42 \%$ de zona rural. Se efectuaron tratamientos en el Hospital Regional consistentes en Crioterapia conización, histerectomía simple o ampliada; según el caso o radioterapia a nivel central. El programa ha posibilitado detectar un gran número de lesiones pre-invasoras, cuyo tratamiento oportuno ha permitido mejorar las condiciones de salud de la población y racionalizar los recursos, demostrándose los beneficios de una atención regionalizada con colposcopia.

Además ha permitido determinar características colpo-citohistológicas y epidemiológicas.

PALABRAS CLAVES: Cuello uterino, cáncer, citología, colposcopia, detección.

\section{Introducción}

Con el propósito de efectuar detección y tratamiento precoz del cáncer del cuello uterino en el Departamento de Caldas Colombia, S.A. se creó un grupo interdisciplinario e interinstitucional conformado por los Departamentos de Ginecoobstetricia y Patología de la Facultad de Medicina, y la Facultad de Enfermería de la Universidad de Caldas, con la cooperación de la Dirección Seccional de Salud de Caldas, Comité Departamental de Cafeteros, Hospital de Caldas y Confamiliares.

El objetivo fundamental del programa ha sido el de disminuir significativamente y a mediano plazo la morbimortalidad producida por esta neoplasia en el Departamento de Caldas, utilizando el recurso de la atención primaria como estrategia para concientizar a la comunidad con el fin de que ésta se movilice y practique el autocuidado.

Para el logro de la meta propuesta, desde junio de 1989 el grupo viaja periódicamente y en forma continua a los

* Facultad de Medicina.

** Facultad de Enfermería. Universidad de Caldas. hospitales regionales del Departamento de Caldas para realizar actividades de prevención, detección, tratamiento y seguimiento del cáncer del cuello uterino. Además realiza actividades educativas al personal de salud en toma e interpretación de la citología cervicouterina, colposcopia, conización, normas de asepsia y procedimientos de quirófanos e igualmente actividades educativas a adolescentes escolares, y comunidades en general, haciendo énfasis a grupos con conductas de riesgo.

El ginecólogo en asocio con los médicos generales de las instituciones practica colposcopia a todas las mujeres a quienes la citología ha sido informada como anormal, con lesiones de bajo grado, alto grado o cáncer invasor.

El programa ha permitido detectar un gran número de tumores en etapa preinvasora o temprana, localizados y relativamente fáciles de curar, evitando así futuras muertes y disminuyendo costos a todas las instituciones de salud.

\section{Materiales y métodos}

Como aplicación del programa descrito se diseño una historia clínica precodificada que se utilizó para recoger la información sobre las características sociodemográficas de 
las pacientes con citología cervicouterina anormal, a quienes se les practicó colposcopia. Incluye además todos los datos con relación a los hallazgos clínicos, colposcópicos, citológicos, histológicos, de tratamiento y seguimiento.

Este estudio se realizó en el Departamento de CaldasColombia, entre los meses de abril de $1989 \cdot y$ junio de 1992. Se practicó colposcopia y biopsia dirigida del cuello uterino a pacientes con lesiones visibles o legrado endocervical a mujeres con colposcopia satisfactoria y zona de transformación típica, sin lesiones excepto a mujeres en embarazo.

La parte del formulario que corresponde a datos de identificación y sociodemográficos de la paciente es diligenciada por una enfermera y la parte clínica del mismo por el médico ginecoobstetra.

El programa está dirigido a atender mujeres en edad fértil, de bajo nivel socioeconómico.

Se analizan varios aspectos:

1. Características Sociodemográficas de las pacientes a quienes se les realizó colposcopia.

2. Proporción de Neoplasia Intraepitelial Cervical (NIC I, II y III) y de cáncer de cuello uterino (microinvasor e invasor) por grupos de edad.

3. Correlación colpocitológica, colpohistológica y citohistológica.

Para los análisis se excluyeron los “sin dato", por tanto los totales varían en las distintas tablas.

Se utilizó programa de computador Epi-Info, aplicando las pruebas de validación estadística correspondientes.

\section{Hallazgos}

Se realizaron 559 colposcopias por primera vez, se diagnosticaron 152 casos de neoplasia intraepitelial y 21 casos de cáncer de cuello uterino en estado avanzado.

\section{Características sociodemográficas}

El promedio de edad de las pacientes que tuvieron cáncer microinvasor o invasor es de 47.7 años. El promedio de edad para las lesiones preinvasoras es de 33.9 años.

El $18.8 \%$ de las pacientes estudiadas tuvieron su primera relación sexual antes de los 15 años y el $79.5 \%$ antes de los 20 años de edad.

\section{Colposcopia}

Se encontraron lesiones atípicas en $65.8 \%$ de los casos, de los cuales el punteado fue la más prevalente seguida del área acetoblanca. En 41 colposcopias se observaron lesiones sospechosas (vasos atípicos o lesiones clínicamente invasoras).

\section{Biopsia}

Se tomaron 404 biopsias, detectándose 21 carcinomas de tipo epidermoide: 4 microinvasores y 17 invasores. Uno de los invasores se detectó en una mujer de 22 años de edad. El $88 \%$ de las lesiones fueron intraepiteliales y se observó asociación entre el estadio de la neoplasia y la edad de la paciente.

\section{CORRELACION COLPO-HISTOLOGICA CALDAS 1989-1992 Biopsia}

\begin{tabular}{|lcc|}
\hline & Anormal & Normal \\
Colposcopia anormal & 160 & 123 \\
Normal & 10 & 106 \\
Sensibilidad $=94.1 \%$ & & \\
Especificidad $=46.3 \%$ & & \\
\hline
\end{tabular}

\section{CARACTERISTICAS SOCIODEMOGRAFICAS DE MUJERES ATENDIDAS CON COLPOSCOPIA SEGUN DIAGNOSTICO FINAL - CALDAS 1989-1992}

\section{EDAD}

\begin{tabular}{|c|c|c|c|c|c|c|c|c|c|}
\hline \multirow{3}{*}{$\begin{array}{l}\text { Variables } \\
\text { Sin dato } \\
0-19\end{array}$} & \multicolumn{2}{|c|}{ Sin dato } & \multicolumn{2}{|c|}{ Cáncer } & \multicolumn{2}{|c|}{ *NIC } & \multicolumn{2}{|c|}{ Normal } & \multirow{3}{*}{$\begin{array}{r}\text { Total } \\
55 \\
26\end{array}$} \\
\hline & 0 & (0) & 1 & (20.0) & 3 & (60.) & 1 & (20.0) & \\
\hline & 6 & (23.1) & 0 & (0) & 4 & (15.4) & 16 & (61.5) & \\
\hline $20-29$ & & (29.8) & 1 & $(0.5)$ & 52 & $(27.7)$ & 79 & $(42.0)$ & 188 \\
\hline $30-39$ & 44 & $(23.0)$ & 5 & (2.6) & 57 & (29.8) & 85 & $(44.5)$ & 191 \\
\hline $40-49$ & 33 & $(33.0)$ & 3 & (3.0) & 28 & $(28.0)$ & 36 & $(36.0)$ & 100 \\
\hline 50-Más & & $(32.7)$ & 11 & (22.4) & 8 & $(16.3)$ & 14 & $(28.6)$ & 49 \\
\hline & & & & & 231 & $(41.3)$ & 559 & & \\
\hline Total & 155 & $(27.7)$ & 21 & (3.8) & 152 & $(27.2)$ & 32.8 & & \\
\hline Promedio & & & 47.7 & & 11.5 & & 9.7 & & \\
\hline Desviac. & & & 11.5 & & 9.3 & & & & \\
\hline & & & & & 9.3 & & & & \\
\hline $\begin{array}{l}\text { Anova una } \\
\text { vía (K- }\end{array}$ & & & & & & & & & \\
\hline Wallis); & & & & & & & & & \\
\hline 27.2; G.L: 2; & & & & & & & & & \\
\hline $\begin{array}{l}\mathrm{P}= \\
0.000001)\end{array}$ & & & & & & & & & \\
\hline
\end{tabular}




\section{Correlación colpohistológica}

De 399 biopsias informadas, 283 presentaban alguna alteración colposcópica y de éstas $160(56.5 \%)$ presentaron algún tipo de lesión histológica que varía desde NIC hasta carcinoma invasor. De 116 colposcopias solamente el $10.3 \%$ presentaron algún tipo de lesión, entre ellas una con carcinoma invasor.

INFORME DE BIOPSIA

Colposcopia NIC1 NIC2 NIC3 MICR Inv Norm Tot.

\begin{tabular}{|lccccccr|}
\hline Acetoblan & 11 & 15 & 44 & 0 & 3 & 41 & 114 \\
Mosaico & 5 & 10 & 32 & 1 & 1 & 22 & 71 \\
Leucoplasia & 2 & 3 & 12 & 0 & 1 & 8 & 26 \\
Punteado & 12 & 18 & 52 & 4 & 9 & 66 & 161 \\
V. Atípicos & 0 & 2 & 9 & 0 & 7 & 2 & 20 \\
\hline
\end{tabular}

La sensibilidad de la colposcopia frente al resultado de la biopsia fue del $94 \%$ y la especificidad del $46.3 \%$.

\section{Correlación colpocitológica}

Sesenta y ocho $(97.1 \%)$ de setenta pacientes con diagnóstico citológico de lesión de alto grado o carcinoma presentaron algún tipo de lesión colposcópica. (Ver tablas anexas).

HALLAZGOS COLPOSCOPICOS EN LAS MUJERES ATENDIDAS - CALDAS 1989-1992

\begin{tabular}{|lrr|}
\hline Hallazgos Colposcópicos & \multicolumn{1}{c|}{ N } & \multicolumn{1}{c|}{$\%$} \\
\hline Vasos atípicos & 27 & 5.3 \\
Cáncer invasor & 14 & 2.8 \\
Area acetoblanca & 161 & 31.3 \\
Mosaico & 86 & 16.7 \\
Leucoplasia & 29 & 5.8 \\
Punteado & 209 & 40.3 \\
Condiloma acuminado & 37 & 7.4 \\
\hline
\end{tabular}

\begin{tabular}{|lcccccccc|}
\hline \multicolumn{1}{|c|}{ EDAD } \\
\hline Informe de \\
Biopsia & Total & $\mathbf{1 9}$ & $\mathbf{2 0 - 2 9}$ & $\mathbf{3 0 - 3 9}$ & $\mathbf{4 0 - 4 9}$ & $\mathbf{5 0 - M a ́ s ~}$ & Prom. & D.E \\
\hline NIC I & 25 & 3 & 8 & 9 & 4 & 1 & 3.5 & 8.9 \\
NIC II & 32 & 1 & 8 & 19 & 3 & 1 & 32.6 & 7.4 \\
NIC III & 92 & 0 & 36 & 29 & 21 & 6 & 35.0 & 9.1 \\
Microinvasor & 4 & 0 & 0 & 1 & 1 & 2 & 48.8 & 14. \\
Invasor & 16 & 0 & 1 & 4 & 2 & 9 & 47.4 & 2 \\
Total & & 169 & 4 & 53 & 62 & 31 & 19 & 11.3 \\
\hline
\end{tabular}

Sin dato $=4$

\section{CORRELACION COLPO-HISTOLOGICA CALDAS 1989-1992}

COLPOSCOPIA

\begin{tabular}{|l|rr|rr|r|}
\hline $\begin{array}{l}\text { Informe de } \\
\text { Biópsia }\end{array}$ & \multicolumn{2}{|c|}{$\begin{array}{c}\text { Satisfactoria } \\
\text { Atípica } \\
\text { Típica }\end{array}$} & \multicolumn{2}{|c|}{$\begin{array}{c}\text { Insatisfactoria } \\
\text { Atípica } \\
\text { Típica }\end{array}$} & Total \\
\hline NIC I & 16 & 3 & 4 & 2 & 25 \\
NIC II & 23 & 2 & 7 & 0 & 32 \\
NIC III & 63 & 2 & 27 & 0 & 92 \\
Microinvas & 2 & 0 & 2 & 0 & 4 \\
OR & 4 & 0 & 12 & 1 & 17 \\
Invasor & & & & & 229 \\
Normal & 92 & 94 & 31 & 12 & 399 \\
Total & 200 & 101 & 83 & 15 & \\
\hline
\end{tabular}

Sin dato $=5$

\begin{tabular}{|l|r|r|c|}
\hline Tratamiento & N & \multicolumn{1}{|c|}{$\%$} & $\begin{array}{c}\% \\
\text { Acumulado }\end{array}$ \\
\hline Observación y control & 137 & $30.9 \%$ & $30.9 \%$ \\
Crioterapia & 32 & $7.2 \%$ & $38.1 \%$ \\
Acido triclordacético & 31 & $7.0 \%$ & $45.0 \%$ \\
Conización & 77 & $17.3 \%$ & $62.4 \%$ \\
Histerectomía & 49 & $11.0 \%$ & $73.4 \%$ \\
Abdominal & 5 & $1.1 \%$ & $74.5 \%$ \\
Histerectomía radical & 9 & $2.0 \%$ & $76.6 \%$ \\
Histerectomía vaginal & 82 & $18.5 \%$ & $95.0 \%$ \\
Repetir colposcopia & 7 & $1.6 \%$ & $96.6 \%$ \\
Radioterapia & 15 & $3.4 \%$ & $100.0 \%$ \\
Otro & & & \\
\hline Total & 44 & $100.0 \%$ & \\
\hline
\end{tabular}


El 42.4\% de la población atendida procede de la zona rural, lo cual refleja el impacto del programa adelantado y que ha tenido por finalidad localizar las pacientes con citología anormal.

El $100 \%$ de la población estudiada es de clase socioeconómica baja, lo cual se correlaciona con la incidencia de la enfermedad en este grupo social (1-3).

Los factores de riesgo descritos como más relacionados con la etiopatogenia de esta neoplasia fueron los mismos que los descritos por Barber 92 (bajo nivel socioeconómico, iniciación sexual a temprana edad y multiparidad) $(1,3)$.

De acuerdo con los resultados de la biopsia se puede deducir que la correlación colpohistológica es buena. Se presentó un caso falso negativo con citología, atribuible a inflamación y necrosis (4-6).

Se efectuaron tratamientos en el hospital regional consistentes en crioterapia, conización, histerectomía simple o ampliada a nivel del hospital local o regional y radioterapia a nivel del hospital de tercer nivel.
El programa ha permitido detectar un gran número de lesiones preinvasoras, que han sido tratadas oportunamente.

Este modelo de trabajo permite la racionalización del recurso humano, tecnológico y científico; aumenta la cobertura y fortalece las políticas de descentralización.

Además ha sido posible unificar terminología, criterios de diagnóstico y terapéuticos entre clínicos y patólogos y determinar las características colpocitohistológicas para mejorar los sistemas de control de calidad en el laboratorio y en un futuro incrementar la especificidad en el diagnóstico citohistológico.

De la sistematización del programa surge un modelo de intervención comunitario en salud que maneja los elementos investigativos como columna vertebral de nuestro trabajo, integración funcional de servicios educativo, asistencial e interdisciplinario. Este modelo puede ser tomado por otros programas de atención en salud y replicado en cualquier contexto.

\section{BIBLIOGRAFIA}

1. Kroeger A., Luna R. Atención primaria para la salud . Principios y Métodos O.P.S. Edit. Pax México 1987.

2. Ministerio de Salud Instituto Nacional de Cancerología. Programa para la detección precoz y control del cáncer del cuello uterino en Colombia, 1990. Santa Fe de Bogotá.

3. Boletín Epidemiológico de Caldas. Programa detección precoz de cáncer de cuello uterino en Caldas. Año 4 No. 3 abril-diciembre de 1990.
4. Coppleson M. Ginecologyc Oncology. Edit Churchil Livingstone Inc. 1 ed. Vol. 1, London 1981.

5. Disaia Philip J., Creasman William T. Ginecología Oncológica. 3 ed. Panamericana 1991

6. Ferency A. Management of the Patient with and abnormal PAP Smear-Conte mporary Obst-Gyn. 1986; 93. 